\title{
REAÇÃO DO MERCADO À ALAVANCAGEM OPERACIONAL: UM ESTUDO EMPÍRICO NO BRASIL*
}

\author{
REACTION OF THE MARKET TO THE OPERATIONAL LEVERAGE: \\ AN EMPIRICAL STUDY IN BRAZIL
}

\author{
JOSÉ ALVES DANTAS \\ Professor Adjunto dos Departamentos de Ciências \\ Contábeis do Centro Universitário UNIEURO e da \\ Universidade Paulista - DF \\ E-mail: alves.dantas@bcb.gov.br

\section{OTÁVIO RIBEIRO DE MEDEIROS} \\ Professor Titular do Departamento de Ciências \\ Contábeis e Atuariais da Faculdade de \\ Economia, Administração, Contabilidade e \\ Ciências da Informação e Documentação da \\ Universidade de Brasília - DF. Professor do \\ Programa Multiinstitucional e Interregional de \\ Pós-Graduação em Ciências Contábeis UnB/ \\ UFPB/UFPE/UFRN \\ E-mail: otavio@unb.br
}

\author{
PAULO ROBERTO B. LUSTOSA \\ Professor Titular do Departamento de Ciências \\ Contábeis e Atuariais da Faculdade de \\ Economia, Administração, Contabilidade e \\ Ciências da Informação e Documentação da \\ Universidade de Brasília - DF. Professor do \\ Programa Multiinstitucional e Interregional de \\ Pós-Graduação em Ciências Contábeis UnB/ \\ UFPB/UFPE/UFRN \\ E-mail: lustosa@unb.br
}

\section{RESUMO}

Estudos que avaliam o impacto de informações contábeis nas variáveis do mercado de ações têm adquirido grande relevância na literatura contábil e se constituído em instrumento de avaliação da utilidade da informação contábil. O presente estudo segue a mesma lógica das pesquisas lucro-retorno, substituindo a medida de resultados contábeis pela de alavancagem operacional e tendo por base os dados das companhias listadas na Bovespa, dos setores de petróleo e gás, materiais básicos, bens industriais, construção e transporte, consumo não cíclico e consumo cíclico, referentes ao período entre o segundo trimestre de 2001 e o terceiro trimestre de 2004. A premissa considerada é a de que, como a alavancagem operacional, além de incorporar uma dimensão de resultado (o lucro operacional), é uma das determinantes do risco sistemático das ações, e de que há relação entre risco e retorno das ações, é possível inferir uma associação positiva entre o grau de alavancagem operacional e o retorno das

\section{ABSTRACT}

Studies evaluating the impact of financial reporting information on capital market variables have gained great importance in the accounting literature and became a tool for assessing the usefulness of accounting information. The present study follows the I path of earnings-return research, measuring operating leverage as a substitute for net income shown in the financial statements. It is based on data between the second quarter of 2001 and the third quarter of 2004 referring to firms listed on Bovespa (São Paulo Stock Exchange) and relating to the following sectors: petroleum and gas, basic materials, industrial goods, construction and transportation, non-cyclical consumption, and cyclical consumption. The hypothesis of the study is that since operating leverage, besides being related to earnings (operating earnings), is one of the factors determining the systematic risk of stocks, and since there is a relationship between risk and stock returns, it is possible to infer a positive relationship between the degree

Recebido em $1^{\circ} .11 .2005$ • Aceito em 23.03.2006

* Artigo originalmente apresentado no XXIX EnANPAD, Brasília-DF, setembro/2005. 
ações. Os testes empíricos realizados com a utilização do método de dados em painel apresentam evidências de que a variável alavancagem operacional é estatisticamente relevante para explicar o comportamento do retorno das ações e que essa relação é positiva, conforme previsto teoricamente. Os resultados, também, demonstram que a relevância estatística aumenta quando são adotados parâmetros mais rigorosos para a consideração dos dados e as conclusões não são determinadas pelo comportamento dos valores extremos (outliers). Os testes de raízes unitárias nas séries e de autocorrelação e heteroscedasticidade nos resíduos reforçam a robustez dos resultados apurados.

Palavras-chave: Alavancagem Operacional; Retorno das Ações; Relação Lucro-Retorno; Utilidade da Informação Contábil. of operating leverage and stock returns. Empirical tests carried out using panel data methods suggest that the operating leverage is statistically relevant in explaining the behavior of stock returns and that this relationship is positive, as predicted by theory. The results also demonstrate that statistical relevance increases when stricter parameters are employed for analyzing the data and that the conclusions are not determined by outlier behavior. Unit root tests on the data series as well as autocorrelation and heteroskedasticity tests on the residuals ensure the robustness of the results obtained.

Keywords: Operating Leverage; Stock Returns; Earnings-Return; Accounting Information Value Relevance.

\section{INTRODUÇÃO}

Desde Ball e Brown (1968), o relacionamento entre informações contábeis e mercados de ações tem sido um dos mais estudados temas na literatura contábil. O forte interesse por esse tipo de pesquisa é legitimado pela previsão teórica de que o provimento de informações relevantes para o processo decisório dos seus usuários, entre eles os investidores, é o objetivo central da Contabilidade. Nesse sentido, tais estudos se concentram em identificar de que forma e em qual dimensão as informações contábeis são efetivamente úteis para o processo decisório dos investidores.

A premissa é que, nas decisões tomadas com o objetivo de maximizar a riqueza, os investidores consideram o impacto de variáveis macroeconômicas e de variáveis específicas na empresa. Como a Contabilidade tem por missão identificar e mensurar essas variáveis específicas, espera-se que as informações contábeis divulgadas sejam consideradas pelo investidor.

$\mathrm{Na}$ literatura, a expressiva maioria desses estudos tem o lucro como a variável contábil examinada. Nesse sentido, cabe ressaltar a afirmação de Martikainen (1993, p.553) de que uma revisão da literatura permite identificar que a relação entre rentabilidade e preço das ações tem sido objeto de um grande número de estudos, mas conhece-se surpreendentemente pouco sobre a relação entre outros indicadores e o preço das ações.
O presente estudo utiliza a mesma lógica teórica da relação lucro-retorno para avaliar a relação entre o retorno e outra variável contábil da empresa, a alavancagem operacional. É presumido que essa variável, por retratar, de certa forma, a eficácia da gestão da estrutura de ativos da organização, se traduz em informação relevante para as decisões de investimentos.

Considerando essa premissa, o objetivo é estruturar um referencial conceitual que dê suporte aos testes empíricos realizados, no sentido de responder à seguinte questão: "no mercado brasileiro, a variável alavancagem operacional é um dos fatores explicativos do comportamento do retorno das ações?"

Os testes empíricos têm por base as companhias listadas na Bolsa de Valores de São Paulo (Bovespa) que integram os setores econômicos ${ }^{1}$ de petróleo e gás, materiais básicos, bens industriais, construção e transporte, consumo não cíclico e consumo cíclico, considerando o período entre o segundo trimestre de 2001 e o terceiro trimestre de 2004.

Além dessa parte introdutória, o trabalho contempla: a revisão da literatura, destacando aspectos conceituais relacionados com a alavancagem operacional, bem como os estudos de associação entre dados contábeis e variáveis de mercado (Seção 2); a apresentação da metodologia utilizada para a realização dos testes empíricos (Seção 3); a apuração e a análise dos resultados (Seção 4) e as conclusões da pesquisa (Seção 5). 


\section{REVISÃO DA LITERATURA}

Uma decisão racional de investimento considera a avaliação de uma série de variáveis que traduzam a situação econômico-financeira corrente da empresa, além de possibilitarem a projeção da viabilidade futura do negócio. Essa análise, ao final, é um dos fatores que permite concluir sobre a possibilidade de agregação de valor para os investidores. Entre essas variáveis, pode-se destacar a alavancagem operacional.

\subsection{Alavancagem Operacional}

O conceito preliminar de alavancagem deriva do significado de alavanca na Física, relacionado com a obtenção de um resultado final mais do que proporcional ao esforço empregado. No ambiente econômico-financeiro das organizações, dois tipos de alavancagem se destacam: a operacional (relacionada com a estrutura de ativos) e a financeira (relativa à estrutura de capitais).

A alavancagem operacional é definida por Garrison e Noreen (2001, p.173) como a medida do grau de sensibilidade do lucro às variações nas receitas de vendas. Traduz a possibilidade de um acréscimo mais do que proporcional no lucro operacional em relação a um aumento nas vendas, funcionando como um efeito multiplicador. Dugan e Shriver (1992, p.310) apresentam uma conceituação mais dinâmica, afirmando que a alavancagem operacional é uma função dos custos fixos e reflete um grau de alteração na estrutura de custos de produção de uma empresa, substituindo custos fixos por variáveis. Em resumo, representa a magnitude da incerteza do lucro operacional em relação à incerteza das vendas.

Verifica-se, assim, que a alavancagem operacional é uma referência utilizada para o gerenciamento dos gastos fixos, cuja otimização é obtida pelo aumento do volume. Essa busca pela otimização é ressaltada por Moyer, McGuigan e Kretlow (1981, p.134), ao afirmarem que, quando uma empresa incorre em custos operacionais fixos, uma ampliação das receitas de vendas resulta em uma mudança relativamente maior no lucro operacional. O que se busca, nessas condições, é a maximização do uso da capacidade instalada (estrutura fixa) da entidade, representada pelos custos e despesas fixas. Assim, se duas empresas possuem o mesmo total de receitas e de despesas, mas têm estruturas de custos diferentes, aquela que apresentar maior proporção de custos fixos terá maior alavancagem operacional.

\subsubsection{O Grau de Alavancagem Operacional (GAO)}

A definição clássica do GAO, medida de expressão da alavancagem operacional, amplamente utilizada nos livros textos, descreve-o como o efeito multiplicador resultante do uso dos custos operacionais fixos, determinado a partir da seguinte equação:

$$
G A O=\frac{x(p-v)}{x(p-v)-F}=\frac{M C}{L O}
$$

em que $x$ representa a quantidade produzida $e$ vendida; $p$ é o preço de venda; $v$ é o custo variável unitário; $F$ representa os custos fixos; $M C$ é a margem de contribuição e $L O$ o lucro operacional.

Essa fórmula de apuração do GAO enfrenta um problema objetivo para sua utilização prática por usuários externos, em função de o sistema de custeio por absorção, utilizado na elaboração das demonstrações contábeis publicadas, não fornecer informações sobre a proporção dos custos fixos e variáveis. Por essa razão, os métodos de estimação do GAO têm assumido um papel de relevância nos trabalhos publicados sobre a alavancagem operacional.

Outra definição, também amplamente divulgada na literatura, segue essa primeira e traduz o GAO como uma medida de elasticidade, tendo em vista que resulta do coeficiente de resposta do lucro operacional em relação a uma mudança no nível das vendas. Percebe-se, assim, que embora o GAO seja uma medida de um período determinado, assume características de dinamismo temporal. Nesse aspecto, é definido da seguinte forma:

$$
G A O=\Delta L O / \Delta R V
$$

em que $\Delta L O$ representa a variação do lucro operacional e $\Delta R V$ significa a variação das receitas de vendas.

De acordo com Lord (1998, p.72), o modelo para estimação do GAO, que tem obtido mais espaço na literatura de finanças, é o utilizado por MandeIker e Rhee (1984). O modelo, baseado em regressão temporal, considera uma derivação da equação (2.2), incorporando o conceito de incerteza. Nessa construção, também referendada por O'Brien e Vanderheiden (1987, p.45) e Dugan e Shriver (1992, p.310), considera-se o conceito mais dinâmico de alavancagem operacional, no sentido de que essa retrata a magnitude da incerteza do lucro operacional em relação à incerteza das vendas. Assim, o GAO é definido como o quociente do desvio do lucro opera- 
cional em relação ao seu valor esperado sobre o desvio das vendas reais em relação ao valor esperado:

$G A O=\left\{\left[\frac{L O_{t}}{E\left(L O_{t}\right)}\right]-1\right\} /\left\{\left[\frac{R V_{t}}{E\left(R V_{t}\right)}\right]-1\right\}$

A partir dessa definição conceitual, Mandelker e Rhee (1984) adotaram um modelo de estimação do GAO que mede a elasticidade do lucro operacional em relação à variação nas vendas, de acordo com a regressão a seguir, em que In é o operador de logaritmo neperiano e o coeficiente de inclinação $-b_{j}$ - fornece uma estimativa da alavancagem operacional da empresa $j$ no período $t$ :

$$
\text { In } L O_{j, t}=a_{j}+b_{j} \operatorname{In} R V_{j, t}+\varepsilon_{j, t}
$$

A partir desse estudo, as pesquisas empíricas envolvendo o grau de alavancagem operacional passaram a utilizar, majoritariamente, esse modelo de estimação, como, por exemplo, Gahlon e Gentry (1986), Huffman (1989), Chung (1989), Dugan, Minyard e Shriver (1994), Darrat e Mukherjee (1995) e Griffin e Dugan (2003).

Essa ampla utilização não resulta em aceitação acadêmica absoluta. O'Brien e Vanderheiden (1987), por exemplo, argumentam que esse modelo apresenta como deficiência a não consideração das possíveis diferenças de tendências de crescimento entre as vendas e o lucro operacional. Também há críticas à utilização de regressões temporais para a estimação do GAO. Dugan, Minyard e Shirver (1994, p.333) discutem essa questão, ao afirmarem que, nesse caso, se assume que os coeficientes de GAO são constantes no período de estimação, tendo em vista que representam a inclinação da regressão linear estimada. Por outro lado, é implícito, na definição teórica da medida de alavancagem operacional, que ela muda no tempo, o que revela uma aparente contradição.

Não obstante as ressalvas de O'Brien e Vanderheiden (1987) e as discussões sobre a adoção de regressões, o modelo sugerido por Mandelker e Rhee (1984) continua como o mais utilizado nos trabalhos empíricos para a estimação do GAO. A relevância de tal modelo é refletida no número de estudos que o replicam e na afirmação de Lord (1998, p.72) de que é o método de estimação do GAO mais popular na literatura de finanças.

\subsubsection{A Alavancagem Operacional e o Risco da Capacidade Instalada}

Toda empresa possui um certo nível de risco, relacionado com a variabilidade inerente às suas atividades ou com a incerteza dos seus resultados, sendo um dos seus componentes o risco associado à sua estrutura fixa. Ao relacionar esse risco à alavancagem operacional, Lev (1974, p.629) destaca que as diferenças de alavancagem operacional entre as empresas se refletirá em diferentes custos unitários variáveis médios e, por sua vez, em níveis distintos de lucro. O autor exemplifica a situação com duas empresas, em que a primeira tem uma alavancagem operacional maior que a segunda, o que se traduz em uma menor proporção dos custos variáveis da primeira em relação à segunda. Assim, a volatilidade dos resultados da primeira empresa, em função de flutuações na demanda, será maior que os da segunda.

Moyer, McGuigan e Kretlow (1981, p.144), também, associam o risco à alavancagem operacional. Os autores demonstram esse relacionamento a partir do seguinte raciocínio: se o GAO é a medida da sensibilidade do lucro da empresa em relação às mudanças no nível das vendas e, assim, quanto maior o GAO da empresa, maior será a mudança no lucro operacional para uma dada alteração nas vendas, então, ceteris paribus, quanto maior o GAO da empresa, maior o nível de risco operacional.

$\mathrm{Na}$ prática, o risco da capacidade instalada materializa-se quando, ao invés do aumento do volume de receitas, ocorre a sua diminuição. Nesse caso, o fenômeno da alavancagem operacional atua em sentido inverso, potencializando as perdas. Moyer, McGuigan e Kretlow (1981, p.143) destacam essa dualidade, afirmando que, dada uma estrutura de GAO elevado, um crescimento das receitas pode resultar em altos ganhos operacionais, do mesmo modo que uma redução pode conduzir a grandes perdas operacionais.

\subsection{A Alavancagem Operacional e o Risco Sistemático das Ações}

Demonstrado que a alavancagem operacional é uma medida contábil que evidencia o risco do negócio, é de se supor que ela tenha relação com o risco sistemático das ações, o beta ( $\beta$ ) do Capital Asset Pricing Model (CAPM), que também é uma medida de risco, só que definida por parâmetros de mercado.

Rubinstein (1973), ao desenvolver uma equação que expressasse o retorno esperado das ações, demonstra, analiticamente, que o prêmio para o risco operacional é o produto de quatro fatores, entre eles a alavancagem operacional, capturado por meio da contribuição marginal ${ }^{2}$. A relação entre o

2 Segundo Gahlon (1981, p.298), esse trabalho foi o primeiro a demonstrar que a alavancagem operacional afeta o risco sistemático. 
risco sistemático e a alavancagem operacional também foi encontrada por Lev (1974), concluindo que, quando a alavancagem operacional cresce (ou decresce), a volatilidade do retorno das ações, também, cresce (ou decresce). As evidências da associação entre essas variáveis foram reforçadas pelas conclusões de Gahlon (1981), demonstrando que as instabilidades observadas nos betas das empresas podem ser explicadas por mudanças no GAO e na medida de variabilidade das vendas.

Incorporando como variáveis explicativas do beta não apenas o GAO, mas também o Grau de Alavancagem Financeira (GAF), Mandelker e Rhee (1984) concluíram que essas variáveis explicam uma grande parcela da variação no beta, sendo positiva e estatisticamente significantes. A repercussão adquirida por esse trabalho decorreu menos da conclusão, que referendou evidências anteriores, e mais da metodologia inovadora adotada pelos pesquisadores para estimar o GAO, conforme já comentado na subseção 2.1.1. Essa relação positiva entre as medidas de alavancagem e risco também foi confirmada por Gahlon e Gentry (1986) e Chung (1989).

Huffman (1989) replicou o trabalho de Mandelker e Rhee (1984), encontrando resultado consistente em relação à alavancagem financeira. No tocante à alavancagem operacional, no entanto, as evidências empíricas só são consistentes com o trabalho anterior quando desconsidera as empresas do segmento de concessionárias de serviços públicos (utilities), o que pode ser derivado, segundo Huffman (1989, p.91), das diferenças extremas na estrutura de capital e no gerenciamento das empresas desse segmento, quando comparado com empresas de outros setores.

Ao formular uma interpretação intuitiva do beta, Dunn (2001, p.34) destacou que o risco sistemático de uma empresa é ampliado pela alavancagem. No caso específico da alavancagem operacional, o autor argumentou que uma típica análise de ponto de equilíbrio mostra como ela influencia a volatilidade, permitindo a conclusão de que empresas com maiores investimentos em ativos têm maior alavancagem operacional, o que tenderá a aumentar seus betas. Nayar e Willinger (2001, p.666), também, destacaram essa associação, a partir do argumento de que uma maior variância nos lucros e fluxos de caixa futuros, relacionada com a alavancagem operacional, aumenta o risco sistemático da empresa, resultando em uma relação positiva entre as duas variáveis de risco.

Griffin e Dugan (2003), igualmente, adotaram o modelo de Mandelker e Rhee, incorporando, além dos riscos operacional e financeiro, o conceito de risco econômico, correspondente a questões de natureza externa à empresa, envolvendo o ambiente político e social, a estrutura do mercado, a posição competitiva da empresa, entre outras. Os testes empíricos revelaram que as três variáveis explicam o beta.

No Brasil, Correia e Amaral (2000) pesquisaram a relação entre o risco sistemático da empresa e o grau de alavancagem operacional, tendo como objeto de estudo o setor siderúrgico, encontrando uma relação linear e positiva entre as variáveis.

\subsection{O Risco e o Retorno das Ações}

Ao analisarem a relação entre os determinantes de risco, entre os quais a alavancagem operacional, e a magnitude dos erros de projeção dos resultados, Comiskey e Mulford (1987, p.30) afirmaram que as conseqüências negativas dos "resultados surpresas" desagradáveis podem ser consideráveis. Assim, freqüentes erros de projeção podem levar ao aumento do risco e, conseqüentemente, ao aumento da taxa de retorno exigida pelos investidores.

Ball, Kothari e Watts (1993) testaram, empiricamente, a associação entre os riscos e os ganhos dos acionistas, comprovando haver uma relação positiva, estatisticamente significativa, entre mudanças nos riscos e nos retornos das ações, reforçando os argumentos de que o risco influencia na determinação do preço das ações.

No mercado de ações brasileiro, a relação entre risco e retorno foi avaliada por Tabak e Guerra (2002), encontrando evidências empíricas que sugerem uma relação positiva e significante entre retornos contemporâneos e volatilidade.

\subsection{As Informações Contábeis e o Retorno das Ações}

A utilidade das informações contábeis sempre mereceu destaque nas discussões teóricas sobre o papel da Contabilidade. A primeira tentativa de estimar a utilidade de números contábeis foi documentada por Ball e Brown (1968), que testaram empiricamente a existência de um relacionamento entre os lucros divulgados e os retornos das ações. Os diversos estudos que os sucederam, com o objetivo de avaliar a relevância da informação contábil, usam uma metodologia comum - exame da associação entre medidas contábeis, geralmente resultados, e valor de mercado. Essa metodologia, segundo Lev e Ohlson (1989, p.251), traduziu-se na forma mais aceita, conceitualmente estruturada e empiricamente testável, de se avaliar a relação entre a informação contábil e seus usos específicos. 


\subsubsection{A Relação Lucro-Retorno}

Considerando que investidores racionais buscam maximizar suas riquezas, Easton (1985, p.54/55) afirma que o preço das ações é, geralmente, associado ao valor presente dos benefícios futuros esperados. Nesse sentido, a associação contemporânea entre lucros contábeis e preço das ações é, então, uma caracterização entre lucros contábeis e benefícios futuros. Assim, a utilização de dados do mercado para testar o conteúdo das informações contábeis, segundo Easton (1985, p.55), não é surpreendente, tendo em vista que os preços das ações são variáveis observáveis, enquanto as variáveis fundamentais - benefícios futuros esperados - são inobserváveis.

Muitas pesquisas têm estudado a relação do mercado com os resultados contábeis. Lev (1989), por exemplo, apresenta uma revisão desses estudos e identifica, apenas no período de 1980 a 1988, no que ele aponta como os três maiores jornais de pesquisa contábil - The Accounting Re- view, Journal of Accounting Research e Journal of Accounting and Economics -, dezenove trabalhos que avaliam diversas combinações de associações de variáveis de retorno e de resultados contábeis. Nichols e Wahlen (2004, p.263) entendem como natural essa concentração de estudos entre lucro e retorno das ações, por tratar-se de duas medidas bottom line de performance da empresa, a primeira de natureza contábil e a segunda do mercado de ações.

Nichols e Wahlen (2004, p.265) apresentam uma interpretação teórica intuitiva para a relação lucro-retorno, a partir de três condições sobre a informação contida no lucro contábil e no preço das ações, nas quais se assume que: (i) o lucro do período corrente disponibiliza informação importante e útil para a projeção de lucros futuros; (ii) as projeções de lucros futuros fornecem importantes elementos para o desenvolvimento das expectativas de dividendos e (iii) o valor presente dos benefícios futuros esperados determina o preço da ação. Tal interpretação está apresentada na figura 1.

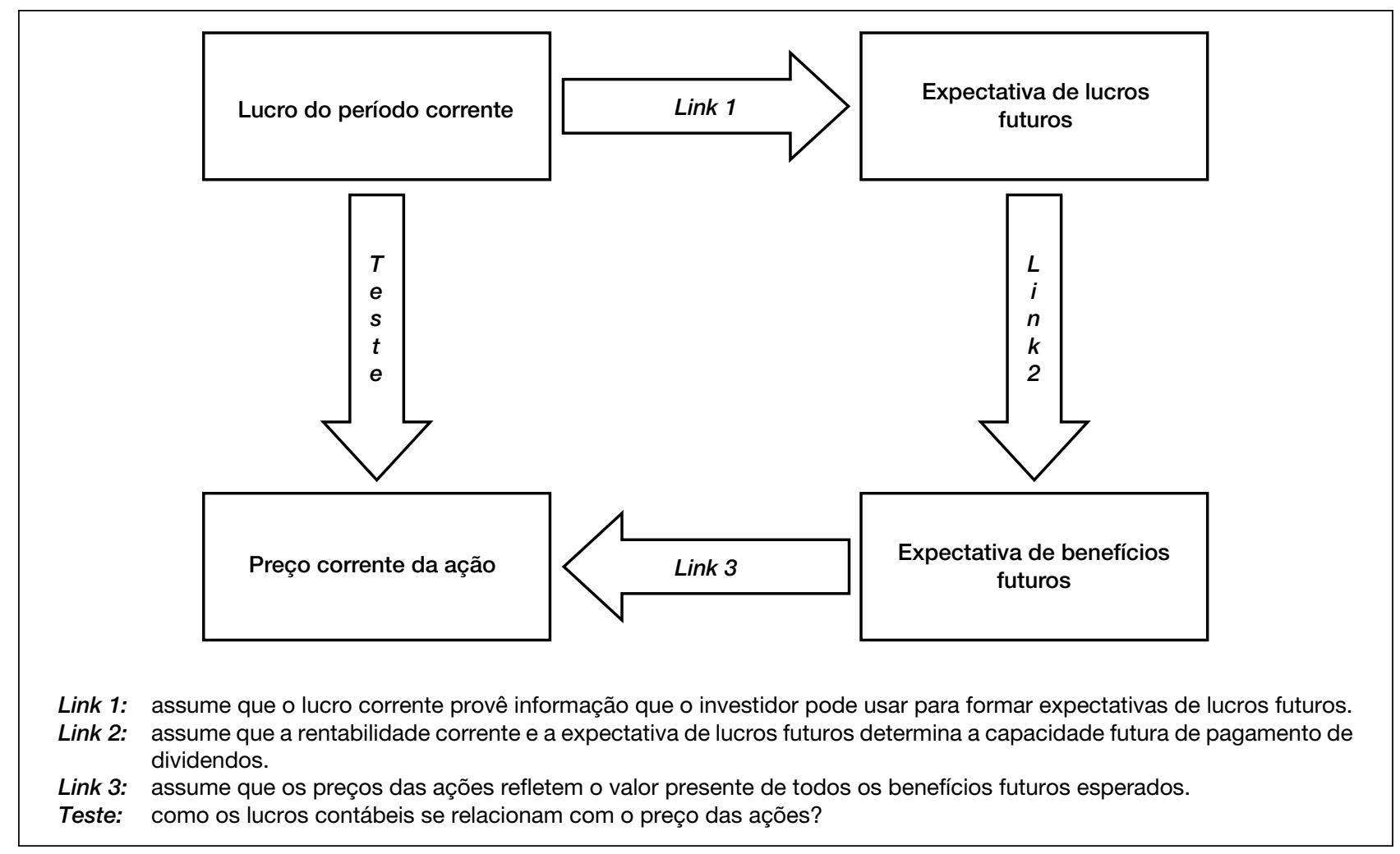

Fonte: Nichols e Wahlen (2004, p.266).

Figura 1 - Links que relacionam lucros contábeis e retorno das ações 


\subsubsection{A Relevância das Parcelas Não Esperadas}

Em relação à discussão sobre a relevância dos lucros contábeis para explicar o comportamento do preço das ações, Lev (1989, p. 158) destaca que algumas pesquisas associam duas medidas de variação: a mudança no preço das ações (retorno) e a mudança no patrimônio da empresa (lucro). O autor argumenta que, considerando que os preços das ações refletem as expectativas sobre resultados futuros antes que esses sejam anunciados, é razoável correlacionar a mudança no preço (retorno) com o lucro não esperado (nova informação) e não com o lucro divulgado.

Esse mesmo entendimento é destacado por Easton (1985, p.75) e Nichols e Wahlen (2004, p.269), ao afirmarem que grande parte da literatura empírica que avalia a associação entre lucros e preço das ações considera a relação entre retornos não esperados (anormal) e lucros não esperados. Isso é considerado por Espahbodi (2000, p.1) como um dos importantes refinamentos estatísticos ocorridos nos estudos que tratam da associação entre essas medidas contábeis e de mercado.

\subsubsection{As Pesquisas sobre a Relação Lucro- Retorno no Brasil}

Conforme evidenciado, os estudos que investigam o impacto da divulgação das informações contábeis no preço das ações, adquiriram, a partir do trabalho de Ball e Brown (1968), relevância na área contábil. No Brasil, no entanto, essa linha de pesquisa ainda pode ser considerada embrionária. Apenas recentemente começa a se fortalecer, com a realização de pesquisas, tendo como objeto de estudo o mercado de ações brasileiro, entre os quais, Mellone (1999), Bernardo (2001), Lopes (2001) e Sarlo Neto et al. (2003).

Embora ainda em um estágio inferior ao verificado na literatura contábil internacional, as evidências encontradas nessas pesquisas, no sentido de que as informações contábeis influenciam o preço das ações no mercado de ações brasileiro, são importantes, tendo em vista que ajudam a dirimir eventuais dúvidas sobre a extensão, ao mercado local, da relação significativamente identificada nos mercados mais desenvolvidos.

\subsection{A Alavancagem Operacional e o Retorno das Ações}

Considerando as associações destacadas nas seções anteriores, entre a alavancagem operacional e o risco da capacidade instalada, entre a alavancagem operacional e o risco sistemático das ações, entre o risco e o retorno das ações e entre os resultados contábeis e o retorno das ações, é possível inferir que a relação entre a alavancagem operacional e o retorno das ações é uma relação teoricamente esperada.

De forma equivalente à relação lucro-retorno, a informação relevante para efeito de se avaliar a repercussão no preço das ações não é o GAO efetivo e sim o GAO não esperado. Isso porque, da mesma forma que no caso do lucro, presume-se que o GAO projetado é incorporado ao preço das ações ex ante. Assim, o que deve provocar alterações no valor das ações é a parcela não prevista da alavancagem operacional.

No que diz respeito ao tipo de reação no preço das ações, em decorrência do GAO não esperado, aguarda-se que essa relação seja positiva, considerando a associação entre risco e retorno das ações. Esse entendimento é reforçado por Martikainen (1993, p.538), ao afirmar que, considerando que existe uma relação teórica positiva entre risco sistemático e alavancagem operacional, deve-se esperar uma relação positiva entre retorno das ações e alavancagem operacional de uma empresa.

\section{METODOLOGIA}

Para a estruturação dos testes, são observadas as seguintes etapas, adaptadas das fases de estudos de eventos citadas por MacKinlay (1997) e Soares, Rostagno e Soares (2002): seleção da amostra, definição do modelo, estimação das variáveis, procedimentos de teste de hipóteses e parâmetros para análise dos resultados.

\subsection{Seleção da Amostra}

A composição da amostra tem por base, além das condições destacadas a seguir, a disponibilidade de dados contábeis das empresas e de preço das ações, referentes ao período considerado, no banco de dados da Economática ${ }^{\circledR}$.

A pesquisa tem como referência as companhias listadas na Bovespa que integram os setores econômicos de petróleo e gás, materiais básicos, bens industriais, construção e transporte, consumo não cíclico e consumo cíclico. A exclusão das companhias do setor de telecomunicações e de utilidade pública fundamenta-se no fato de que esses setores passaram, no período considerado na pesquisa (1995 a 2004), por profunda reestruturação, decorrente do processo de privatização e de definições de marco regulatório, o que poderia influenciar as medidas de retorno de forma distinta do mercado 
e distorcer as conclusões da pesquisa. A exclusão das instituições financeiras, por sua vez, justifica-se em função das características específicas dessas empresas, cujas estruturas de ativos e de capitais são próprias, não podendo ser tratadas dentro dos mesmos parâmetros das demais organizações, sob risco de distorção dos resultados empíricos.

Tendo em vista o modelo utilizado para a estimação do GAO, especificado na seção 3.4 , são incorporadas na amostra apenas as empresas que apresentem seqüência de resultados operacionais positivos. Isso porque a estimação é realizada a partir de regressão em que uma das variáveis consideradas é o logaritmo natural do lucro operacional, o que impõe a necessidade do resultado positivo.

Das empresas que atendam a essas condições, são excluídas da amostra as ações que apresentem, entre 2000 e 2004, menos de cem dias de negociação por ano, em média. A justificativa é de que a inclusão de ações com pouca liquidez pode provocar distorções nos resultados da pesquisa, em função de não guardarem, necessariamente, relação com o comportamento do mercado.

Tendo por base as condições estabelecidas em relação aos dados das empresas e à negociação das ações, a amostra final considerada para a realização da pesquisa é integrada por 81 combinações empresa/ação ${ }^{3}$. Os testes empíricos têm como referência os parâmetros trimestrais de alavancagem operacional, entre janeiro de 2001 e junho de 2004, e de retorno, entre abril de 2001 e setembro de 2004. Essa defasagem temporal entre as variáveis se justifica pela premissa destacada na seção anterior, que relaciona o retorno não esperado de determinado período com o GAO não esperado do período imediatamente anterior. Os dados contábeis de 1995 a 2000 e os de retorno de abril de 2000 a março de 2001 são utilizados para permitir a projeção das variáveis, de acordo com os modelos a serem destacados a seguir.

\subsection{Definição do Modelo Econométrico}

A aplicação dos testes empíricos envolve a especificação de uma regressão linear, a partir da qual se pode verificar a relação entre a alavancagem operacional e o retorno das ações. Na elaboração do modelo econométrico, são consideradas as premissas destacadas na seção 2.5 , de que as mudanças nos preços das ações devem ser relacionadas com o GAO não esperado e não com o GAO divulgado. Seguindo esse raciocínio, o modelo assume a seguinte forma:

$R N E_{i j, t}=\alpha_{i j}+\beta_{i j}\left(G A O N E_{i j, t-1}\right)+\varepsilon_{i j, t}$

em que $R N E_{i j}$ representa o retorno não esperado da ação $i$ da empresa $j$, no período $t$, e $G A O N E_{i, t-1}$ é o GAO não esperado da empresa $j$, titular da ação $i$, no período $t-1$.

Para os efeitos do presente estudo, os retornos das ações e os graus de alavancagem operacional são considerados em bases anualizadas, assim entendidos por observarem um período de doze meses (não se confundindo, necessariamente, com o ano civil), o que reduz os riscos de distorções decorrentes de fatores de sazonalidade. Essa preocupação se justifica, principalmente, pelo fato de a alavancagem operacional estar relacionada com o nível de utilização da capacidade de produção, que, por sua vez, é influenciada por aspectos sazonais.

Tendo em vista que os dados contábeis são divulgados trimestralmente, os retornos e GAO são reavaliados na mesma periodicidade, configurando um novo período de referência para a realização dos testes empíricos. Dessa forma, no período $t+1$, os dados contábeis e de retorno do primeiro trimestre do período $t$ serão substituídos pelos do novo trimestre.

Outra questão a ser observada é que a associação estudada pressupõe uma janela de tempo para que os efeitos do GAO não esperado se reflitam no retorno das ações, razão pela qual o retorno não esperado acumulado durante o período $t$ é relacionado com o GAO não esperado referente ao período $t-1$.

\subsection{Estimação do Retorno Não Esperado (RNE)}

O primeiro passo para a mensuração do retorno não esperado é o estabelecimento da sistemática

\footnotetext{
3 Petrobras/PN, Petrobras/ON, Ipiranga Ref/PN, Ipiranga Dist/PN, Ipiranga Pet/PN, Caemi/PN, Vale Rio Doce/PN, Vale Rio Doce/ON, Acesita/PN, Acesita/ON, Belgo Mineira/PN, Belgo Mineira/ON, Cosipa/PN, Cosipa/ON, Ferbasa/PN, Gerdau/PN, Gerdau/ON, Sid Nacional/ON, Sid Tubarão/PN, Usiminas/PNA, Usiminas/ON, Confab/ PN, Mangels/PN, Paranapanema/PN, Braskem/PN, Copesul/ON, Polialden/PN, Unipar/PNB, Adubos Trevo/PN, Fertibrás/PN, Fosfertil/PN, Rhodia-Ster/ON, Duratex/PN, Aracruz/PNB, Aracruz/ON, Ripasa/PN, Suzano/PN, Votorantim Cel/PN, Klabin/PN, Magnesita/PNA, Embraer/PN, Embraer/ON, Albarus/ON, Fras-le/PN, Marcopolo/PN, Metal Leve/PN, Randon Part/PN, Iochp-Maxion/PN, Embraco/PN, Mundial/PN, Inds Romi/PN, Metisa/PN, Forjas Taurus/PN, Weg/PN, Itautec/ON, Sultepa/PN, Avipal/ON, Sadia/PN, Seara/PN, Seara/ON, Bunge Brasil/PN, Bunge Brasil/ON, Perdigão/PN, Ambev/PN, Ambev/ON, Souza Cruz/ON, Bombril/PN, Pão de Açúcar/PN, Coteminas/PN, Pettenati/PN, Santista Textil/PN, Teka/PN, Arthur Lange/PN, Alpargatas/PN, Saraiva Livr/PN, Net/PN, Guararapes/PN, Guararapes/ON, Globex/PN, Lj Americanas/PN, Lj Americanas/ON.
} 
de apuração dos retornos das ações. Para tal fim, é adotado o método de capitalização contínua, que possibilita uma maior robustez em seus resultados, quando comparado com o método de capitalização discreta, conforme argumentam Brooks (2002, p.7) e Soares, Rostagno e Soares (2002, p.5).

$R_{i, t}=\operatorname{In}\left(p_{i, t} / p_{i, t-1}\right)=\operatorname{In} p_{i, t}-\operatorname{In} p_{i, t-1}$

em que $R_{i, t}$ é o retorno da ação $i$, no período $t$, enquanto $p_{i, t}$ e $p_{i, t-1}$ se referem ao preço ${ }^{4}$ da ação $i$, nos momentos $t$ e $t-1$, respectivamente.

O passo seguinte consiste em definir o retorno não esperado $(R N E)$, determinado por:

$$
R N E_{i, t}=R_{i, t}-E_{t-1}\left(R_{i, t}\right)
$$

em que $R_{i, t}$ é o retorno apurado de acordo com a equaçấo (3.2) e $E_{t-1}\left(R_{i, t}\right)$ representa o retorno esperado da ação $i$ para o período $t$, no tempo $t-1$.

Para a apuração do $R N E$, resta estabelecer a metodologia para a definição do retorno esperado das ações. É adotado o modelo estatístico de cálculo de retorno anormal, em que o retorno é ajustado ao risco e ao mercado. Esse modelo assume, segundo Soares, Rostagno e Soares (2002, p.7), que os retornos anormais das ações são observados pela divergência dos retornos individuais efetivamente ocorridos em relação ao retorno do portfolio de mercado. Assim, o retorno esperado é apurado a partir de uma regressão linear entre os retornos diários da ação e a variação diária do índice de mercado, de acordo com a seguinte equação:

$$
E_{t-1}\left(R_{i, t}\right)=\hat{\alpha}_{i}+\hat{\beta}_{i} R_{m, t}
$$

em que $R_{m, t}$ representa o retorno do mercado (lbovespa) no período $t$, enquanto $\hat{\alpha}_{i}$ e $\hat{\beta}_{i}$ são os coeficientes para o período estimado, tendo por referência os dados do período $t-1$.

Seguindo essa construção, o retorno não esperado apurado estará ajustado ao risco e ao mercado, reduzindo a possibilidade de os resultados serem influenciados pelo comportamento do mercado, o que enviesaria as conclusões da pesquisa.

\subsection{Estimação do Grau de Alavancagem Operacional Não Esperado (GAONE)}

Para a mensuração da variável explicativa do modelo, o GAONE, a primeira dificuldade é a apu- ração do GAO, em função da não divulgação dos dados segregados sobre custos fixos e variáveis, condição para se apurar o GAO pelo método convencional. Como forma de suprir essa deficiência, é adotado o modelo desenvolvido por Mandelker e Rhee (1984) e utilizado por Gahlon e Gentry (1986), Huffman (1989), Chun (1989), Dugan, Minyard e Shriver (1994), Darrat e Mukherjee (1995) e Griffin e Dugan (2003), definido da seguinte forma:

$$
\operatorname{In} L O_{j, t}=a_{j}+b_{j} \operatorname{In} R O L_{j, t}+\varepsilon_{j, t}
$$

O coeficiente de inclinação da linha de regressão, $b_{i}$, traduz o GAO da empresa $j$, no período $t$, tendo em vista que representa a variação do lucro operacional que é explicada pela variação nas receitas operacionais líquidas.

Definida a forma de estimação do GAO, pode-se adotar raciocínio equivalente ao utilizado no processo de apuração do retorno não esperado - equação (3.3) -, para a determinação do GAO não esperado, empregando-se a seguinte equação:

$$
G A O N E_{j, t}=G A O_{j, t}-E_{t-1}\left(G A O_{j, t}\right)
$$

em que $\mathrm{GAO}_{j, t}$ é o GAO apurado de acordo com o modelo (3.5) - coeficiente $b_{j}-\mathrm{e} E_{t-1}\left(G A O_{j, t}\right)$ é o GAO esperado para o período $t$, no momento $t-1$.

Também de forma equivalente ao cálculo do $R N E$, a apuração do GAONE depende da projeção do GAO esperado. Lev e Ohlson (1982, p.259) ressaltam que a ênfase em dados contábeis não esperados leva a uma limitação intrínseca da metodologia, em virtude da necessidade de assumir um modelo específico de expectativa.

Em relação à definição dessa proxy, é utilizada como referência a previsão de que as propriedades temporais da relação lucro-retorno demonstra que os resultados contábeis seguem um passeio aleatório (random walk), conforme afirmam Espahbodi (2000, p.1) e Babalyan (2002, p.17). Isso pressupõe que uma unidade monetária de "surpresa" nos lucros do ano $t$ resulta em uma revisão de mesmo montante na projeção dos resultados para o ano $t+1$. Babalyan (2001, p.17) é ainda mais específico, ao afirmar que, baseado na hipótese de que os lucros contábeis seguem um passeio aleatório, a diferença entre o lucro do período $t$ e o do período $t-1$, a chamada primeira diferença, é definida como lucro não esperado. Nichols e Wahlen (2004, p.269), por sua vez, afirmam que pesquisadores e participantes 
do mercado de ações, geralmente, usam projeções consensuais de analistas como proxy para a expectativa do mercado. Alternativamente, os autores admitem que se as projeções dos analistas não estão disponíveis, o que é o caso da presente pesquisa, os dados dos períodos anteriores, geralmente, servem como uma estimativa dos valores esperados.

Utilizando-se, por analogia, esse método de projeção do lucro, conhecido como naive model (modelo ingênuo), o GAO esperado para o período $t$ é definido, neste trabalho, como o GAO de $t-1$, conforme equação a seguir:

$$
E_{t-1}\left(G A O_{j, t}\right)=G A O_{j, t-1}
$$

Fica claro que a utilização desse critério de mensuração para o GAO esperado considera dois tipos de limitação: primeiro, o pesquisador não pode observar diretamente as expectativas dos investidores em relação a essa variável no futuro; segundo, o mercado brasileiro não dispõe de um instituto que consolide e divulgue as projeções dos analistas para os exercícios seguintes, o que permitiria a utilização de tais informações como referência para a dimensão esperada da variável contábil, no caso o GAO.

\subsection{Procedimentos de Teste das Hipóteses}

Os testes empíricos, baseados no modelo econométrico (3.1), têm como objetivo avaliar a relevância estatística da variável independente, GAONE, para explicar o comportamento da variável dependente, RNE. Nesse sentido, são testadas as seguintes hipóteses, nula e alternativa:

$H_{0}$ : O GAO não explica o comportamento crosssectional do retorno não esperado das ações.

$H_{l}$ : O GAO explica o comportamento cross-sectional do retorno não esperado das ações.

Para a realização dos testes, o modelo econométrico desenvolvido é aplicado considerando a metodologia de dados em painel ou panel data, que considera o comportamento das variáveis tanto na dimensão temporal quanto na espacial. Baltagi (2001, p.5-7) e Marques (2000, p.1-2) apresentam uma série de vantagens da estimação com dados em painel, entre as quais podem ser destacadas: o controle da heterogeneidade individual; o provimento de dados com maior poder de informação, maior variabilidade, menor colinearidade entre as variáveis, maior número de graus de liberdade e maior eficiência estatística na estimação; a possibilidade de se facilitar uma análise mais eficiente das dinâmicas de ajustamento; a possibilidade de serem identificados e medidos efeitos que não são detectáveis em estudos exclusivamente seccionais ou temporais, entre outras.

Associando-se esses benefícios às características da pesquisa, em que são considerados aspectos seccionais (amostra composta de diferentes combinações empresa/ação) e temporais (comportamento do grau de alavancagem operacional e do retorno das ações ao longo de determinado período), fica evidenciada a relevância da utilização dos dados em painel para aumentar a eficiência estatística dos testes realizados neste estudo.

Considerando a dinâmica do presente estudo, em que o objetivo é avaliar se a parcela não esperada do GAO é um dos elementos que explicam o comportamento do retorno anormal das ações, são utilizados os três modelos de dados em painel: sem efeitos $^{5}$ (pooled regression), com efeitos fixos e com efeitos aleatórios. Com isso, pretende-se verificar se a hipótese nula da pesquisa pode ser rejeitada, independentemente do modelo utilizado.

Para aferir a robustez dos resultados empíricos, são realizados testes quanto à existência de raízes unitárias nas séries, tendo por objetivo verificar a sua estacionariedade, evitando o problema das regressões espúrias, e quanto à existência de autocorrelação e de heteroscedasticidade nos resíduos das regressões, com a finalidade de avaliar se são atendidas as hipóteses do modelo clássico de regressão linear, condição para que os estimadores possam ser considerados como Best Linear Unbiased Estimators (BLUE).

\subsection{Parâmetros para Análise dos Resultados}

A análise dos resultados considera, preliminarmente, que se a hipótese nula, $\mathrm{H}_{0}$, for rejeitada, a variável GAONE influencia o comportamento cross-sectional do RNE das ações. Nesse caso, os resultados corroboram a hipótese alternativa, $\mathrm{H}_{1}$, respondendo afirmativamente à questão-problema destacada na parte introdutória do presente estudo e permitindo concluir-se que "no mercado brasileiro, a variável grau de alavancagem operacional é um dos elementos informativos do comportamento do retorno das

50 modelo sem efeitos pressupõe que tanto o intercepto quanto as inclinações não variam. Não considera, dessa forma, a heterogeneidade dos dados, razão pela qual Marques (2005, p.5) entende que sua aplicação não é verdadeiramente um método de estimação em painel. 
ações". A não rejeição da hipótese nula, por sua vez, leva a conclusões em sentido contrário.

\section{APURAÇÃO E ANÁLISE DOS RESULTADOS}

Utilizando-se como referência os modelos descritos e a periodicidade trimestral, são apuradas as variáveis de retorno (retorno real, retorno esperado e retorno não esperado) e de alavancagem operacional (GAO efetivo, GAO esperado e GAO não esperado) de cada combinação empresa/ação integrante da amostra. Uma questão a ser destacada na apuração dessas variáveis é a distinção entre a determinação do valor efetivo do retorno das ações e do GAO em determinado período. No caso do retorno, a medida apurada é concreta e observável. Em relação ao GAO, no entanto, tendo em vista que as demonstrações contábeis não especificam os custos fixos e variáveis, a apuração do GAO é dada por estimativa.

Concluído o processo de apuração das medidas de retorno e de alavancagem operacional, cujo objetivo principal foi a determinação das variáveis $R N E$ e GAONE, são realizados os testes de associação entre essas duas variáveis, condição fundamental para se concluir sobre os objetivos da pesquisa, tendo como referência o modelo (3.1).
Na realização desses testes, são utilizados os três modelos de estimação de dados em painel efeitos constantes (pooled regression), efeitos fixos e efeitos aleatórios - e considerados três procedimentos de cálculos:

- Procedimento 1: incorpora todas as combinações de RNE e GAONE disponíveis, sem levar em consideração se, na estimação do GAO, esse se revelou conceitualmente inconsistente $^{6}$ (valores negativos) ou estatisticamente não significativo (estatística $t$ irrelevante);

- Procedimento 2: exclui as observações que foram derivadas dos dados de GAO inconsistentes do ponto de vista conceitual ou estatisticamente irrelevantes;

- Procedimento 3: exclui as observações com valores extremos (outliers) - distantes além de três desvios padrões, para mais ou para menos, em relação à média.

\subsection{Testes de Associação RNE × GAONE}

Para se apurar a relevância da variável GAO$N E$ para explicar o comportamento da variável $R N E$, foram utilizados os dados de acordo com os três procedimentos de cálculo e os três modelos de estimação de dados em painel. A tabela 1 apresenta um resumo dos resultados apurados:

Tabela 1 - Resumo dos Testes de Associação RNE $\times$ GAONE

\begin{tabular}{|c|c|c|c|c|c|c|}
\hline \multicolumn{7}{|c|}{$\begin{array}{l}\text { Variável dependente: RNE } \\
\text { Variável independente: GAONE } \\
\text { Período considerado: 2001:2 2004:3 } \\
\text { Número de observações incluídas: } 14 \text { trimestres }\end{array}$} \\
\hline Proced. & Obs. no Painel & Modelo & Estatística t & p-valor & R2 & R2 Ajustado \\
\hline \multirow[t]{3}{*}{ Proc. 1} & 1.096 & Sem efeitos & 3,759725 & 0,0002 & 0,012756 & 0,011854 \\
\hline & & Efeitos fixos & 3,085582 & 0,0021 & 0,130339 & 0,060869 \\
\hline & & Efeitos aleatórios & 3,515904 & 0,0005 & 0,099308 & 0,098484 \\
\hline \multirow[t]{3}{*}{ Proc. 2} & 1.001 & Sem efeitos & 4,278230 & 0,0000 & 0,017992 & 0,017009 \\
\hline & & Efeitos fixos & 3,002178 & 0,0027 & 0,111251 & 0,033969 \\
\hline & & Efeitos aleatórios & 3,755186 & 0,0002 & 0,089791 & 0,088880 \\
\hline \multirow[t]{3}{*}{ Proc. 3} & 971 & Sem efeitos & 3,981265 & 0,0001 & 0,016094 & 0,015079 \\
\hline & & Efeitos fixos & 3,140077 & 0,0017 & 0,127322 & 0,048879 \\
\hline & & Efeitos aleatórios & 3,586281 & 0,0004 & 0,110521 & 0,109603 \\
\hline
\end{tabular}


As estatísticas $t$ mostradas, na tabela, revelam que a variável independente, GAONE, é estatisticamente relevante para explicar o comportamento da variável independente, RNE, independentemente do procedimento de cálculo considerado e do modelo de panel data adotado. O p-valor apurado em todas as situações, também, reforça a relevância da relação entre as duas variáveis, revelando a significância estatística sempre acima de $99 \%$.

Comparando-se os resultados apurados nos procedimentos 1 e 2 , verifica-se que o segundo apresenta estatísticas $t$ mais relevantes quando utilizados os modelos de efeitos constantes e de efeitos aleatórios, enquanto no modelo de efeitos fixos se constata uma redução. Cabe ressaltar que nos dois modelos que apresentaram variação positiva, essa se deu em uma dimensão muito mais relevante que no caso do modelo em que a estatística sofreu redução. Com isso, é possível afirmar-se que a relevância da estatística $t$ aumenta ao se adotar critérios mais rigorosos de estimação da medida de alavancagem operacional.

A incorporação do procedimento 3 tem como objetivo avaliar a consistência e robustez da associação entre as duas variáveis, a partir do exame do impacto dos valores extremos. Isso porque, em alguns casos, a dimensão dos outliers pode ser determinante na de- finição do comportamento médio do conjunto, o que poderia enviesar os resultados. Comparando-se as estatísticas $t$ desse procedimento com as do 2 , verifica-se o aumento da relevância da associação quando utilizado o modelo de efeitos fixos e a redução quando se utilizam os modelos sem efeitos e o com efeitos aleatórios. Quando comparadas com o procedimento 1 , as estatísticas $t$ do procedimento 3 são mais relevantes, independentemente do modelo utilizado.

A análise dos dados dos coeficientes de determinação - $R^{2}$ - demonstra a importância do método de dados em painel. Enquanto o modelo sem efeitos, que não considera a heterogeneidade dos dados da amostra, não incorporando, portanto, a principal vantagem da especificação estatística dos dados em painel, apresenta um coeficiente de determinação sempre abaixo de $2 \%$, os modelos de efeitos fixos e de efeitos aleatórios apresentam $R^{2}$ muito mais relevantes, em torno de $10 \%$.

\subsection{Testes de Raízes Unitárias das Séries}

Os resultados dos testes de raízes unitárias - ADF Fisher e PP Fisher - das séries, para os três procedimentos de cálculo considerados nos testes de associação entre as variáveis RNE e GAONE, são consolidados na tabela 2.

Tabela 2 - Resumo dos Testes de Raízes Unitárias das Séries

\begin{tabular}{c|c|c|c|c|c|c|c}
\multirow{2}{*}{$\begin{array}{c}\text { Tipo de } \\
\text { Teste }\end{array}$} & \multirow{2}{*}{$\begin{array}{c}\text { Série } \\
\text { (variável) }\end{array}$} & \multicolumn{2}{|c|}{ Proc. 1 } & \multicolumn{2}{c|}{ Proc. 2 } & \multicolumn{2}{c}{ Proc. 3 } \\
\cline { 3 - 8 } & Estatística & p-valor & Estatística & p-valor & Estatística & p-valor \\
\hline ADF - Fisher & RNE & 592,386 & 0,0000 & 556,420 & 0,0000 & 539,483 & 0,0000 \\
\hline & GAONE & 405,954 & 0,0000 & 338,131 & 0,0000 & 389,176 & 0,0000 \\
\hline PP - Fisher & RNE & 689,814 & 0,0000 & 669,217 & 0,0000 & 624,578 & 0,0000 \\
\hline & GAONE & 351,284 & 0,0000 & 369,106 & 0,0000 & 326,480 & 0,0000 \\
\hline
\end{tabular}

O confronto dos valores das estatísticas dos testes com os da tabela de distribuição qui-quadrada confirmam que as séries não apresentam raízes unitárias, independentemente do cenário considerado. Isso permite afirmar que as regressões não são espúrias, configurando-se, assim, em um dos elementos da robustez dos resultados encontrados.

\subsection{Testes de Autocorrelação dos Resíduos}

Com o objetivo de verificar a existência de autocorrelação nos resíduos, foram realizados testes Durbin-Watson para os três diferentes procedimentos de cálculo e os três tipos de modelos de dados utiliza- dos, cujos resultados são consolidados na tabela 3.

Dos nove testes realizados, em sete não há evidências de autocorrelação. Os outros dois se situam em área indefinida, não permitindo concluir-se pela presença ou ausência de autocorrelação nos resíduos. Cabe ressaltar que esses dois testes se referem ao modelo de efeitos constantes, menos relevante para as conclusões da pesquisa, pelo fato de não considerar a heterogeneidade dos dados: principal vantagem apresentada pelo método de dados em painel. Dessa forma, é possível concluir-se que os testes quanto à existência de autocorrelação nos resíduos reforçam a robustez dos resultados empíricos da pesquisa. 
Tabela 3 - Resumo dos Testes Durbin-Watson de Autocorrelação dos Resíduos

\begin{tabular}{c|l|c|l}
\hline Procedimento & Modelo de panel data & Durbin-Watson Stat & Resultado do teste \\
\hline Procedimento 1 & Sem efeitos & 1,736938 & Inconclusivo \\
\hline & Efeitos fixos & 1,971883 & Não há evidências de autocorrelação \\
\hline & Efeitos aleatórios & 1,903879 & Não há evidências de autocorrelação \\
\hline Procedimento 2 & Sem efeitos & 1,825959 & Não há evidências de autocorrelação \\
\hline & Efeitos fixos & 2,018613 & Não há evidências de autocorrelação \\
\hline & Efeitos aleatórios & 1,970364 & Não há evidências de autocorrelação \\
\hline Procedimento 3 & Sem efeitos & 1,722913 & Inconclusivo \\
\hline & Efeitos fixos & 1,942466 & Não há evidências de autocorrelação \\
\hline & Efeitos aleatórios & 1,905789 & Não há evidências de autocorrelação \\
\hline
\end{tabular}

\subsection{Testes de Heteroscedasticidade dos Resíduos}

Para verificar a existência de heteroscedasticidade nos resíduos, foi utilizada a versão de Bickel (1978) para o teste de Breusch-Pagan, em que as probabilidades observam uma distribuição $F$. Os resultados dos testes realizados são consolidados na tabela 4 , a seguir.
Os testes revelaram a presença de heteroscedasticidade nos resíduos no procedimento 1 , enquanto nos procedimentos 2 e 3 a hipótese nula de homocedasticidade não é rejeitada, concluindo-se pela ausência de heteroscedasticidade nos resíduos. Em relação a esses resultados, cabe ressaltar que o procedimento 1 considera todas as combinações disponíveis das variáveis RNE e GAONE, inclusive aquelas decorrentes de GAO conceitualmente

Tabela 4 - Resumo dos Testes Breusch-Pagan de Heteroscedasticidade dos Resíduos (Versão de Bickel)

\begin{tabular}{c|c|l}
\hline Procedimento & $\mathbf{F}$ - Stat $(\mathbf{p}=5)$ & \multicolumn{1}{c}{ Resultado do teste } \\
\hline Procedimento 1 & 9,373287 & Hipótese nula de homocedasticidade é rejeitada \\
\hline Procedimento 2 & 2,987820 & Hipótese nula de homocedasticidade não é rejeitada \\
\hline Procedimento 3 & 1,189962 & Hipótese nula de homocedasticidade não é rejeitada \\
\hline
\end{tabular}

inconsistente ou estatisticamente não significativos. Dessa forma, o cenário 1 - o único que acusou a presença de heteroscedasticidade -, não pode ser considerado como relevante para as conclusões da pesquisa, tendo em vista as restrições dos dados considerados.

Nos procedimentos 2 e 3 - em que são consideradas apenas as observações derivadas de GAO que, no processo de estimação, se mostraram conceitualmente consistentes e estatisticamente relevantes - não foram identificados sinais de heteroscedasticidade nos resíduos, o que se traduz em mais um elemento de robustez dos resultados da pesquisa.

\section{CONCLUSÕES}

Em função do exposto ao longo do trabalho e tendo por referência os objetivos definidos na parte introdutória, a primeira conclusão que se pode afirmar é quanto à sustentação teórica da presumida associação positiva entre a alavancagem operacional e o retorno das ações. A premissa teórica demonstrada é a de que como a alavancagem operacional, além de incorporar uma dimensão de resultado (lucro operacional), é uma das medidas determinantes do risco sistemático das ações e de que há relação entre risco e retorno das ações, é possível inferir-se uma associação entre o grau de alavancagem operacional e o retorno das ações.

Evidenciada a sustentação teórica da relação avaliada, o passo seguinte consistiu na realização dos testes empíricos, considerando-se como variáveis no modelo final do teste de associação, as parcelas não esperadas do retorno e do GAO, partindo-se do pressuposto de que os preços das ações refletem as expectativas futuras.

Os resultados dos testes realizados, considerando-se a combinação dos dados apresentados 
nos três tipos de procedimentos de cálculos com os três tipos de modelos de dados em painel, evidenciaram que, em qualquer combinação procedimento/modelo, a variável independente - GAONE - é estatisticamente relevante para explicar parte do comportamento da variável dependente - RNE.

Adicionalmente, uma análise mais detalhada dos resultados permite concluir que as evidências tornam-se mais robustas ao se adotarem critérios mais rigorosos de estimação da medida de alavancagem operacional, à medida que as estatísticas $t$ dos testes de associação do procedimento 2 são, em geral, mais relevantes que as apresentadas nos testes do procedimento 1. A incorporação do procedimento 3, por sua vez, com a exclusão dos outliers, demonstra que a significância estatística da variável explicativa não é determinada pelo comportamento dos valores extremos, o que aumenta a relevância dos resultados empíricos encontrados.

Os testes quanto à existência de raízes unitárias nas séries e de autocorrelação e heteroscedasticidade dos resíduos, por sua vez, demonstraram que as séries não possuem raízes unitárias (as regressões não são espúrias) e que não há evidências de autocorrelação e de heteroscedasticidade nos termos de perturbação (os estimadores podem ser considerados BLUE), o que reforça a robustez dos resultados empíricos.

Em decorrência dos testes realizados, é possível concluir pela rejeição da hipótese nula da pesquisa, o que resulta na afirmação de que o GAONE é estatisticamente significante para explicar o RNE das ações. Corrobora, assim, a hipótese alternativa do estudo e responde afirmativamente à questão-problema destacada na parte introdutória do estudo, concluindo que "no mercado brasileiro, a variável grau de alavancagem operacional é um dos elementos informativos do comportamento do retorno das ações".

Embora não seja fundamental para se concluir sobre a questão da pesquisa, a análise do $\mathrm{R}^{2}$ serve para demonstrar a relevância da utilização dos dados em painel. Isso porque os testes com modelos sem efeitos, em que não são consideradas as heterogeneidades individuais dos elementos da amostra, o coeficiente de determinação é sempre bem inferior ao apresentado nos testes com modelos de efeitos fixos ou de efeitos aleatórios.

Entre as limitações, há que se ressaltar, que as conclusões do presente estudo não podem ser generalizadas a todos os segmentos com ações negociadas na Bovespa, tendo em vista que não incluiu todos os setores do mercado de ações brasileiro. Outra limitação deve-se ao fato de que não há, no mercado brasileiro, instituições ou banco de dados que sistematizem as previsões de indicadores (para o caso do presente estudo, retorno e GAO), o que resulta na necessidade de se estimar um modelo de previsão, que não necessariamente reflita adequadamente e com precisão as estimativas dos agentes de mercado.

\section{REFERÊNCIAS BIBLIOGRÁFICAS}

BABALYAN, Levon. Association between accounting earnings and stock returns as a measure of value relevance of accounting standards: empirical evidence from the swiss market. EFMA 2002 London Meetings, 2002. Disponível em <http://papers.ssrn.com/ sol3/>. Acesso em 23 jul., 2004.

BALL, Ray; BROWN, Philip. An empirical evaluation of accounting income numbers. Journal of Accounting Research, vol. 6, $\mathrm{n}^{\circ}$ 6, p.159-178, 1968.

; KOTHARI, S. P.; WATTS, Ross W.. Economic determinants of the relation between earnings changes and stock returns. The Accounting Review, vol. 68, n³, p.622-638, 1993.

BALTAGI, B. H.. Econometric analysis of panel data. 2 ed. West Sussex, UK: John Wiley, 2001.

BERNARDO, H. P.. Avaliação empírica do efeito dos anúncios trimestrais do resultado sobre o valor das ações no mercado brasileiro de capitais - um estudo de evento. 2001. Dissertação (Mestrado em Ciências Contábeis) - Departamento de Contabilidade e Atuaria - FEA/USP, SP.

BICKEL, P. J.. Using residuals robustly I: tests for heteroscedasticity, nonlinearity. The Annals of Statistics, vol. $6, n^{\circ} 2$, p.266291, 1978.
BROOKS, Chris. Introductory econometrics for finance. Cambridge: Cambridge University Press, 2002.

CHUNG, Kee H.. The impact of the demand volatility and leverages on the systematic risk of common stocks. Journal of Business Finance \& Accounting, vol. 16, n 3, p.343-360, 1989.

COMISKEY, Eugene E.; MULFORD, Charles W.. Risk determinants and the accuracy of utilities' earnings forecasts. Public Utilities Fortnightly, vol. 119, n 4, p.30-34, 1987.

CORREIA, Laíse F. C.; AMARAL, Hudson F.. A relação entre o risco sistemático da empresa e o grau de alavancagem operacional: análise do setor siderúrgico brasileiro. Anais do XXIV EnANPAD. Rio de Janeiro: ANPAD, 2000. CD-ROM.

DARRAT, Ali F.; MUKHERJEE, Tarun K.. Inter-industry differences and the impact of operating and financial leverages on equity risk. Review of Financial Economics, vol. 4, n² 2, p.141-155, 1995.

DUGAN, Michael T.; SHRIVER, Keith A.. An empirical comparison of alternative methods for the estimation of the degree of operating leverage. The Financial Review, vol. 27, n² 2, p.309-321, 1992.

; MINYARD, Donald H.; SHRIVER, Keith A.. A reexamination of the operating leverage-financial leverage tradeoff 
hypothesis. The Quarterly Review of Economics and Finance, vol. 34, n 3, p.327-334, 1994.

DUNN, Michael F.. An intuitive interpretation of beta. Proceedings of the Academy of Economics and Economic Education, vol. 4, $\mathrm{n}^{\circ}$ 1, p.33-36, 2001.

EASTON, Peter D. Accounting earnings and security valuation: empirical evidence of the fundamental links. Journal of Accounting Research, vol. 23, p.54-77, 1985.

ESPAHBODI, Reza. On the factors influencing return-earnings relationship. Working Paper Series, 2000. Disponível em <http:// papers.ssrn.com/sol3/>. Acesso em 23 jul., 2004.

GAHLON, James M.. Operating leverage as a determinant of systematic risk. Journal of Business Research, vol. 9, p.297-308, 1981.

; GENTRY, James A.. On the relationship between systematic risk and the degrees of operating and financial leverage. Financial Management, vol. 11, n² 2, p.15-23, 1986.

GARRISON, Ray H.; NOREEN, Eric W. Contabilidade gerencial. 9 ed., Rio de Janeiro: LTC Editora, 2001.

GRIFFIN, Harry, F.; DUGAN, Michael T.. Systematic risk and revenue volatility. The Journal of Financial Research, vol. $26, n^{\circ} 2$, p.179-189, 2003

HUFFMAN, Stephen P.. The impact of the degrees of operating and financial leverage on the systematic risk of common stocks: another look. Quarterly Journal of Business and Economics, vol. $28, \mathrm{n}^{\circ} 1, \mathrm{p} .83-100,1989$.

LEV, Baruch. On the association between operating leverage and risk. The Journal of Financial and Quantitative Analysis, vol. 9, $\mathrm{n}^{\circ}$ 4, p.627-641, 1974.

On the usefulness of earnings and earnings research: lessons and directions from two decades of empirical research. Journal of Accounting Research, vol. 27, supplement, p.153-192, 1989

; OHLSON, James A.. Market-based empirical research in Accounting: a review, interpretation, and extension. Journal of Accounting Research, vol. 20, supplement, p.249-322, 1989.

LOPES, Alexsandro B.. A relevância da informação contábil para o mercado de capitais: o modelo de Ohlson aplicado à Bovespa. 2001. Tese (Doutorado em Ciências Contábeis) - Departamento de Contabilidade e Atuária, FEA/USP, SP

LORD, Richard A.. Properties of time-series estimates of degree of leverage measures. The Financial Review, vol. 33, p.69-84, 1998.

MACKINLAY, A.. Events studies in Economic and Finance. Journal of Economic Literature, v. 35, n 1, p.13-39, 1997.

\section{Endereço dos Autores:}

Centro Universitário UNIEURO

Av. das Nações, trecho 0, cj.5 - Campi Brasília

Brasília-DF

70.200-001

Universidade Paulista

SGAS Quadra 913, s/nº - cj. B - Asa Sul - Campi Brasília

Brasília - DF

70390-130
MANDELKER, Gershon N.; RHEE, S. Ghon. The impact of the degrees of operating and financial leverage on systematic risk of common stock. The Journal of Financial and Quantitative Analysis, vol. 19, n 1 , p.45-57, 1984.

MARQUES, Luis David. Modelos dinâmicos com dados em painel: revisão de literatura. Centro de Estudos Macroeconomicos e Previsão - Faculdade de Economia do Porto, Outubro de 2000. Disponível em <http://fep.up.pt/investigacao/workingpapers>. Acesso em 26 jan., 2005

MARTIKAINEN, Teppo. Stock returns and classification pattern of firm-specific financial variables: empirical evidence with finish data. Journal of Business Finance \& Accounting, vol. $20, \mathrm{n}^{\circ} 4$, p.537-558, 1993.

MELLONE, Geraldo. Evidência empírica da relação cross-section entre retorno. Anais do XXIII EnANPAD. Foz do Iguaçú: ANPAD, 1999. CD-ROM.

MOYER, R. Charles; McGUIGAN, James R.; KRETLOW, William J.. Contemporary financial management. St. Paul: West Publishing, 1981.

NAYAR, Nandkumar; WILLINGER, G. Lee. Financial implications of the decision to increase reliance on contingent labor. Decision Sciences, vol. 32, n 4, p.661-681, 2001.

NICHOLS, D. Craig; WAHLEN, James M.. How do earnings numbers relate to stock returns? A review of classic accounting research with updated evidence. Accounting Horizons, vol. 18, $\mathrm{n}^{\circ}$ 4, p.263-286, 2004.

O'BRIEN, Thomas J.; VANDERHEIDEN, Paul A.. Empirical measurement of operating leverage for growing firms. Financial Management, vol. 16, $\mathrm{n}^{\circ}$ 2, p.45-53, 1987.

RUBINSTEIN, Mark E.. A mean-variance synthesis of corporate financial theory. The Journal of Finance, vol. 28, $n^{\circ}$ 1, p.167-181, 1973.

SARLO NETO, Alfredo; et al.. Uma investigação sobre a capacidade informacional dos lucros contábeis no mercado acionário brasileiro. Anais do $3^{\circ}$ Congresso USP Controladoria e Contabilidade. São Paulo (SP): USP, 2003. CD-ROM.

SOARES, Rodrigo O.; ROSTAGNO, Luciano M.; SOARES, Karina T. C.. Estudo de evento: o método e as formas de cálculo do retorno anormal. Anais do XXVI EnANPAD. Salvador: ANPAD, 2002. CD-ROM.

TABAK, B. Miranda; GUERRA, Solange M.. Stock returns and volatility. Working Paper Series BCB n.54, 2002. Disponível em <http:www.bcb.gov.br>. Acesso em 16 set., 2004.

Universidade de Brasília

Faculdade de Econ., Adm., Contab. e Ciência da Inform. e Documentação

Campus Darcy Ribeiro - Prédio da FACE

Asa Norte - Brasília - DF

70910-900 